\title{
LA TIPIFICACIÓN DE MALVA SPICATA L. (MALVACEAE)
}

\author{
por ANTONIO KRAPOVICKAS y CARMEN L. CRISTOBAL
}

\section{Summary}

The specimen 870.1 (LINN) is proposed as lectotype of Malva spicata L. The figure «Sloan. jam. t. 138, f. 1 ", mentioned in the protologue by Linnaeus, is incompatible with the position of Malva spicata in Monadelphia polyandria and in the genus Malva L. of the linnean system, so its exclusion from the protologue is confirmed in accordance with K. Schumann (1886) and Fawcett \& Rendle (1926).

Fryxell (1988: 457) publica la nueva combinación Melochia spicata (L.) Fryxell, Sterculiaceae, basada en Malva spicata L. (1759a: 1146).

En el protólogo de Malva spicata Linnaeus dice: «M. fol. cordatis crenatis tomentosis, spicis oblongis hirtis. Sloan. jam. t. 138, f. 1». La descripción es muy breve, no permite una identificación precisa, y no coincide con ninguna de las frases usadas por Sloane (1707). La lámina de Sloane representa a Melochia villosa (Mill.) Fawc. et Rendle.

Fawcett y Rendle (1926: 106) interpretan Malva spicata L., con el ejemplar 870.1. LINN, «named by Linnaeus Malva spicata». En este caso, como para la mayoría de las especies lineanas de Malváceas, no emplean la palabra «type», que sin embargo usan con frecuencia en el resto de la obra, por lo cual no se podría tomar como una formal tipificación. Hay que tener en cuenta que el método del tipo nomenclatural, recién se incorpora en forma expresa en las Reglas de Nomenclatura de 1935 y que Fawcett fallece en 1926, el año de edición de las Malváceas en la Flora de Jamaica.

Según Fawcett y Rendle (1926: 106), al tratar Malvastrum spicatum (L.) A. Gray, Malva spicata L. abarca dos elementos: el ejemplar 870.1. LINN y Sloane, Jam. t. 138. f. 1 (Sloane, 1707).

Tanto Fawcett \& Rendle como Schumann (1886: 46) excluyen expresamente la lámina de

'Instituto de Botánica del Nordeste(UNNE-CONICET). C.C. 209. 3400 Corrientes, Argentina.
Sloane, que Fawcett \& Rendle identifican como Melochia villosa (Mill.) Fawc. \& Rendle y Schumann como Meiochia hirsuta Cav. (= Melochia villosa).

Borssum Waalkes (1966: 155) afirma que Malva spicata L. fue basada solamente en la lámina (t. 138, f. 1) de Sloane, que designa como holotipo.

El mismo Borssum Waalkes (1966: 155), definitivamente propone Malvastrum spicatum (L.) A. Gray, como lectotipo del género Malvastrum, lo que prueba que este autor, interpretó erróneamente la lámina de Sloane, que sin dudas es una Melochia, y citada como tal por Schumann y por Fawcett y Rendle.

Hill (1982: 196-97) señala que la t. 138, f.1 de Sloane ilustra a Melochia villosa y que en el Sloane Herbarium (BM) hay un ejemplar de Malvastrum americanum anotado como «Althaea spicata», considerado como parte del protólogo y elegido como lectotipo. Sin embargo esta elección no es válida ya que Linnaeus no estudió el herbario Sloane y basó sus especies solamente en las ilustraciones o descripciones de Sloane (Stearn, 1957: 120).

Sobre la base de la tipificación de Borssum Waalkes solamente, Fryxell (1988: 457) hace la nueva combinación Melochia spicata (L.) Fryxell, que de acuerdo a la interpretación de Fawcett y Rendle no corresponde aplicar al género Melochia.

Una evidencia de que Linnaeus tuvo en sus manos una planta, es que ubicó su Malva spicata, en Monadelphia Polyandria, como corres- 
ponde al ejemplar 870.1. LINN. Si se hubiese basado únicamente en la lámina de Sloane, difícilmente habría apreciado los caracteres florales por los cuales Malva se diferencia de Melochia que el mismo Linnaeus ubica en Monadelphia Pentandria. En otras palabras, la lámina por si sola es incompatible con la ubicación de esta planta en el sistema.

En el mismo año Linnaeus (1759b, 1760: 401) publica la disertación de Elmgren sobre una colección de plantas de P. Browne, de Jamaica, que Linnaeus adquirió en 1758. En ella amplía la descripción de Malva spicata, atribuyéndole un doble cáliz, carácter de Malva («Cal. duplex») pero no de Melochia.

De acuerdo con el Código de Nomenclatura (1994, Recommendation 9A.5), «When two or more heterogeneous elements were included in or cited with the original description or diagnosis, the lectotype should be so selected as to preserve current usage. In particular, if another author has already segregated one or more elements as other taxa, the residue or part of it should be designated as the lectotype provided that this element is not in conflict with the original description or diagnosis».

Con la elección del ejemplar 870-1 LINN como lectotipo se preserva el concepto que el mismo Linnaeus aplicó a Malva spicata, claramente expresado por su anotación del nombre en el lectotipo aquí propuesto, y por su ampliación de la descripción realizada en el mismo año. También se preserva la interpretación de Schumann (1886) y el criterio de Fawcett \& Rendle (1926), quienes excluyen la ilustración de Sloane («excl. reference to Sloane»).

De esta manera se evita la publicación de nuevas combinaciones para Melochia villosa var. regnellii (K. Schum.) A. Goldberg y var. tomentosa (K. Schum.) A. Goldberg.

De acuerdo a esta interpretación la sinonimia sería la siguiente:

\section{Malvastrum americanum (L.) Torrey}

Torrey, Rep. U.S. Mex. Bound. 38, 1859.

Malva americana L. Sp. pl. 687, 1753. Tipo: Hortus

Leydenensis, A. van Royen s.n. (L 908.139-311)

(lectotipo designado por Borssum Waalkes, 1966: 155).

Malva spicata L. Syst. nat. ed. 10, 2: 389, 1759. Lectotipo: 870.1 LINN!, aquí designado.

Malvastrum spicatum (L.) A. Gray. Mem. Amer. Acad. Arts n.s. 4: 22, in adn., 1849.

Malveopsis americana (L.) Kuntze. Rev. gen. pl. 1: 72, 1891 .

Malvecpsis spicata (L.) Kuntze. Rev. gen. pl. 1:72, 1891.

Sphaeralcea americana (L.) Metz, Catholic Univ. Amer., Biol. Ser. 16: 142, 1934.

Melochia spicata (L.) Fryxell. Syst. Bot. Mon. 25: 457, 1988.

\section{Bibliografía}

BORSSUM WAALKES, J. van. 1966. Malesian Malvaceae revised. Blumea 14: 1-213.

FAWCETT, W. \& A.B. RENDLE. 1926. Fl. Jamaica 5. Malvastrum: 104-106.

FRYXELL, P. A. 1988. Malvaceae of Mexico. Syst. Bot. Mon. 25: 1-522.

GREUTER, W. 1994. Chairman Editorial Comittee. International code of botanical nomenclature. Regnum Veg. 131.

HILL, S. R. 1982. A monograph of the genus Malvastrum A. Gray (Malvaceae: Malveae). Rhodora 84 (837): 1-83, (838): 159-264, (839): 317-409.

LINNAEUS, C. 1759a. Syst. nat. ed. 10, 2: 1146 (mai-june 1759).

- 1759b. Fl. jamaic. pug. 32 pgs. (28 nov. 1759). Descripción de plantas presentadas en 1758 por Patrick Browne a Linnaeus.

- 1760. Pugillus jamaic. plant. Amoen. acad. 5 (97): 389413 'Sep. 1760). (Nueva edición de Linnaeus, 1759b).

SCHUMANN, K. 1886. Sterculiaceae en Martius Fl. bras. 12(3): 1-114.

SLOANE, H. 1707. A voyage to the Islands Madera, Barbados, Nieves, S. Christopher and Jamaica. vol. 1. London.

STEARN, W. T. 1957. Introduction to C. Linnaeus, Sp. pl. Facsimile first edition 1753. 1: The Sloane herbarium pg. 119-120. 